\title{
Differences in cancer stage, treatment and in-hospital mortality between patients with and without schizophrenia: retrospective matched-pair cohort study
}

Hanako Ishikawa, Hideo Yasunaga, Hiroki Matsui, Kiyohide Fushimi and Norito Kawakami

\section{Background}

Healthcare access and outcomes in cancer patients with schizophrenia remain unclear.

\begin{abstract}
Aims
To investigate the likelihood of early diagnosis and treatment in patients with schizophrenia who have cancer and their prognosis.

\section{Method}

A retrospective matched-pair cohort of gastrointestinal cancer patients was identified using a national in-patient database in Japan. Multivariable ordinal/binary logistic regressions was modelled to compare cancer stage at admission, invasive treatments and 30-day in-hospital mortality between patients with schizophrenia $(n=2495)$ and those without psychiatric disorders $(n=9980)$.
\end{abstract}

\section{Results}

The case group had a higher proportion of stage IV cancer (33.9\% v. 18.1\%), a lower proportion of invasive treatment ( $56.5 \%$ v. $70.2 \%$, odds ratio $(\mathrm{OR})=0.77,95 \% \mathrm{Cl} 0.69-0.85)$ and higher in-hospital mortality $(4.2 \%$ v. $1.8 \%, \mathrm{OR}=1.35,95 \% \mathrm{Cl}$ 1.04-1.75).

\section{Conclusions}

Patients with schizophrenia who had gastrointestinal cancer had more advanced cancer, a lower likelihood of invasive treatment and higher in-hospital mortality than those without psychiatric disorders.

\section{Declaration of interest}

None.

\section{Copyright and usage}

(c) The Royal College of Psychiatrists 2016.
Life expectancy of people with schizophrenia has been reported to be shorter than that of the general population ${ }^{1-3}$ and the gap has increased. ${ }^{4,5}$ Most excess deaths in the schizophrenia population have been reported to be as a result of chronic physical conditions, such as cardiovascular disease and cancer. ${ }^{2,5-10}$ Studies indicated that the cancer incidence in patients with schizophrenia was no higher than in the general population. ${ }^{11-17}$ However, cancer mortality in patients with schizophrenia was higher than in non-psychiatric patients. ${ }^{10,18-21}$ The reason for the discrepancy between cancer incidence and mortality in patients with schizophrenia remains unclear; ${ }^{20}$ however, potential related factors may include delayed diagnosis and lower likelihood of receiving cancer care. Several studies have found a difference in access to cancer care between patients with schizophrenia and the general population, ${ }^{22}$ with those with schizophrenia being less likely to receive screening for cancer. ${ }^{23-26} \mathrm{~A}$ few studies suggested that people with schizophrenia may have undergone delays in the diagnosis of cancer and initiation of treatment. ${ }^{18,27,28}$ Also, some studies indicated that patients with schizophrenia were less likely to receive standard surgical and non-surgical treatment for cancer. ${ }^{18,27,28}$ However, most studies have been limited owing to a lack of detailed patient background data, including cancer stage and comorbidity. The only study to include information about cancer staging measured the presence of metastases, which is rather crude compared with cancer stage. ${ }^{18}$ Further, many studies have had small sample sizes and lacked control groups. Additionally, little is known about the difference in short-term mortality following treatment between patients with schizophrenia and non-psychiatric patients. ${ }^{29}$ In the present study, we hypothesised that patients with schizophrenia were less likely to receive an early diagnosis of cancer, less likely to undergo invasive cancer treatments and more likely to have a poor prognosis. To test these hypotheses, we used a national in-patient database in Japan to conduct a matched-pair cohort study: we compared cancer stage on admission, receipt of surgical and endoscopic treatments and 30-day in-hospital mortality between gastrointestinal cancer patients with schizophrenia and those without psychiatric disorders.

\section{Method}

\section{Data source}

The Japanese Diagnosis Procedure Combination database is a national database of in-patients in Japan. Details of the database have been described elsewhere. ${ }^{30,31}$ In brief, the database contains administrative claims and discharge abstract data including the following: unique hospital identifiers; postal codes of patient residential areas; patients' age and gender; diagnoses and comorbidities on admission coded according to ICD-10; procedures; tumour, node, metastasis classification of malignant tumours and cancer stage; pack-year smoking status; and in-hospital death. As of 2012, the database included approximately 7 million in-patients from around 1000 hospitals, representing approximately $50 \%$ of all acute care in-patient admissions in Japan. Approval for this study was obtained from the Institutional Review Board at The University of Tokyo. Because of the anonymous nature of the data, the requirement for informed consent was waived.

\section{Patient selection and data}

We identified patients aged $\geqslant 40$ years who were admitted to the participating hospitals with a primary diagnosis of gastric cancer (ICD-10 code, C16) or colorectal cancer (C18-20) and were discharged between 1 July 2010 and 31 March 2013. We excluded 
patients with recurrent cancer. Cancer stage was categorised into four subgroups (stages I-IV). Patients with missing data on cancer stage were defined as the 'missing' group. For non-psychiatric comorbidities, each ICD-10 code for a comorbidity was converted into a score based on Quan's algorithm, ${ }^{33}$ and these were totalled for each patient to calculate the Charlson comorbidity index (CCI).$^{34}$ Because all patients had a malignancy diagnosis (CCI score 2), the summed CCI scores were divided into three categories $(2,3-4$ and $\geqslant 5)$. Smoking status was classified into three groups: non-smoker, smoker and unknown status.

For the patients' socioeconomic status, we used the average household income per capita in the municipality where the patient lived. These data were derived from the Statistical Observations of Municipalities 2012 (www.stat.go.jp/english/). The average neighbourhood income per capita for each patient was categorised into quintiles. The reason for admission was divided into three groups based on the diagnosis prior to admission: cancer, schizophrenia and other.

\section{Matching}

Among the patients with gastrointestinal cancer, we selected the case group (patients with schizophrenia) and the control group (patients without any psychiatric disorder) by means of 1:4 matching. Patients with a diagnosis of schizophrenia were identified using ICD-10 code F20-29. The control group were those without any diagnosis of psychiatric disorders (F00-F99). For each person in the case group, we identified a set of control patients who were in the same age category $(40-49,50-59,60-69,70-79$ and $80-89$ years old) and were admitted to the same institution in the same year and month. We then randomly selected four controls per individual in the case group from the pooled population of controls.

\section{Analyses}

We compared patient characteristics between the case and the control groups. We also compared the distribution of the cancer stage on admission between the two groups. We then compared the proportions of patients undergoing surgical or endoscopic treatment and their length of stay as well as 30-day in-hospital mortality between the groups stratified by cancer stage (I-IV). Because the data-set does not include post-discharge information, we used in-hospital death within 30 days after admission in our analyses. We performed all these comparisons using chi-squared tests.

In the matched groups, we performed multivariable ordinal logistic regression to analyse the difference in cancer stage on admission between the case and control groups - with adjustment for age, gender, CCI, smoking status, site of cancer (gastric or colorectal) and average neighbourhood income per capita. We conducted multivariable binary logistic regression analysis to compare the receipt of surgical or endoscopic treatment between the case and control groups - with adjustment for age, gender, CCI, smoking status, site of cancer (gastric or colorectal), average income per capita and cancer stage (I-IV). We carried out multivariable binary logistic regression analysis to compare 30-day in-hospital mortality between the case and control groups - with adjustment for age, gender, CCI, smoking status, site of cancer (gastric or colorectal), average income per capita, cancer stage (I-IV), receipt of surgical or endoscopic treatment and reason for admission. Generalised estimating equations were used to account for clustering within each set of five patients (i.e. one in

\begin{tabular}{|c|c|c|c|c|c|}
\hline & \multicolumn{2}{|c|}{ Case group $(n=2495)$} & \multicolumn{2}{|c|}{ Control group $(n=9980)$} & \multirow[b]{2}{*}{$P$} \\
\hline & $n$ & $\%$ & $n$ & $\%$ & \\
\hline Gender & & & & & 0.002 \\
\hline Male & 1546 & 62.0 & 6521 & 65.3 & \\
\hline Female & 949 & 38.0 & 3459 & 34.7 & \\
\hline Age, years & & & & & 1.000 \\
\hline $40-59$ & 156 & 6.3 & 624 & 6.3 & \\
\hline $60-69$ & 803 & 32.2 & 3212 & 32.2 & \\
\hline 70-79 & 1102 & 44.2 & 4408 & 44.2 & \\
\hline$\geqslant 80$ & 434 & 17.4 & 1736 & 17.4 & \\
\hline Charlson comorbidity index score & & & & & $<0.001$ \\
\hline$\leqslant 2$ & 864 & 34.6 & 4316 & 43.2 & \\
\hline $3-4$ & 852 & 34.1 & 3438 & 34.4 & \\
\hline$\geqslant 5$ & 779 & 31.2 & 2226 & 22.3 & \\
\hline Smoking status & & & & & 0.007 \\
\hline Non-smoker & 1373 & 55.0 & 5340 & 53.5 & \\
\hline Smoker & 767 & 30.7 & 3372 & 33.8 & \\
\hline Unknown & 355 & 14.2 & 1268 & 12.7 & \\
\hline Average neighbourhood income, thousand US dollars & & & & & 0.872 \\
\hline$<10.8$ & 450 & 18.0 & 1854 & 18.6 & \\
\hline $10.8-12.2$ & 463 & 18.6 & 1864 & 18.7 & \\
\hline $12.3-13.5$ & 563 & 22.6 & 2159 & 21.6 & \\
\hline $13.6-15.8$ & 484 & 19.4 & 1965 & 19.7 & \\
\hline$>15.8$ & 508 & 20.4 & 2049 & 20.5 & \\
\hline Data missing & 27 & 1.1 & 89 & 0.9 & \\
\hline Type of cancer & & & & & 0.213 \\
\hline Gastric cancer & 1265 & 50.7 & 5199 & 52.1 & \\
\hline Colorectal cancer & 1230 & 49.3 & 4781 & 47.9 & \\
\hline Reason for admission & & & & & $<0.001$ \\
\hline Treatment for cancer & 2318 & 92.9 & 9639 & 96.6 & \\
\hline Treatment for schizophrenia & 11 & 0.4 & 0 & 0.0 & \\
\hline Other & 166 & 6.7 & 341 & 3.4 & \\
\hline
\end{tabular}


the case group and four in the control group). We set the statistical significance level at 0.05 for a two-sided test. All analyses were performed using SPSS version 22.0.

\section{Results}

\section{Patient characteristics}

We identified 436170 patients with primary gastrointestinal cancer during the survey period. Among them, we found 4660 (1.1\%) patients with schizophrenia and 412617 (94.6\%) nonpsychiatric patients. Of these 417277 patients, 182716 patients had gastric cancer, 209915 colorectal cancer and 24646 both gastric and colon cancer. Using 1:4 matching, we selected 2495 individuals with schizophrenia (the case group) and 9980 nonpsychiatric controls (the control group). The 2165 excluded patients in the case group had fewer than four counterparts in the control pool. Table 1 presents the demographic data. The case group had a higher CCI and a larger proportion of stage IV cancer than the control group. No significant difference was seen in the average neighbourhood income per capita. The proportion of patients who were admitted for cancer treatment was slightly higher in the control group (96.6\%) than in the case group (92.9\%).

\section{Cancer stage on admission}

The proportion of stage I cancer patients in the case group was smaller than that in the control group (23.8\% v. 43.6\%), and the proportion of stage IV cancer patients in the case group was greater than in the control group $(33.9 \%$ v. $18.1 \%, P<0.001)$. Data on the cancer stage was missing in $170(6.8 \%)$ of those in the case group and $730(7.3 \%)$ in the control group (Table 2).

Table 3 presents the results of the multivariable ordinal logistic regression analysis for cancer stage fitted with a generalised estimation equation. After adjusting for patients' gender, age, CCI, average neighbourhood income, smoking status and type of cancer, the case group was significantly more likely to have a higher cancer stage than the control group: relative risk, 1.86, 95\% CI 1.72-2.00; $P<0.001$.

\section{Undergoing surgical or endoscopic treatment and length of stay}

The proportion of patients receiving surgical or endoscopic treatment was significantly different between the two groups: $56.5 \%$ in the case group and $70.2 \%$ in the control group, $P<0.001$ (Table 2). Regarding type of invasive treatment, those in the case group were more likely to receive open surgery $(34.0 \%)$ than the non-psychiatric control group (29.5\%). The control group were more likely to undergo laparoscopic surgery or endoscopic treatment than patients with schizophrenia ( $14.5 \%$ v. $18.4 \%, 7.9 \%$ v. $22.3 \%$, respectively). When stratified by cancer stage, the proportions of patients undergoing surgical or endoscopic treatment for stage I cancer were $84.5 \%$ and $88.0 \%$ respectively in the case and control groups $(P=0.024)$. The respective proportions for stage IV cancer were $20.4 \%$ and $31.7 \%(P<0.001)$.

The median length of stay was 25 days (interquartile range $(\mathrm{IQR})=15-43)$ for the case group and 15 days $(\mathrm{IQR}=9-24)$ for the control group (Table 2). Multivariable binary logistic regression analysis fitted with a generalised estimation equation showed that the case group was significantly less likely to undergo surgical or endoscopic treatment than the control group - odds ratio $(\mathrm{OR})=0.77,95 \%$ CI $0.69-0.85, \quad P<0.001$ - even after adjustment for cancer stage (Table 4 ).

\section{0-day in-hospital mortality}

The case group had higher 30-day in-hospital mortality (4.2\%) than the control group $(1.8 \%, P<0.001)$ (Table 2). Table 5 shows the results of multivariable binary logistic regression analysis for 30-day mortality fitted with a generalised estimation equation. After adjusting for all the patients' characteristics, the case group had significantly higher mortality than the control group $(\mathrm{OR}=1.35 ; 95 \%$ CI 1.04-1.75, $P=0.026)$ - even after adjustment for cancer stage, undergoing invasive treatments and reason for admission. Being older, having an advanced cancer stage and higher CCI, and not receiving invasive treatments were also significantly associated with 30-day in-hospital mortality.

\section{Discussion}

\section{Main findings}

The present retrospective matched-pair cohort study using a national in-patient database in Japan found that patients with gastrointestinal cancer and schizophrenia were more likely to have advanced cancer on admission than those without psychiatric disorders. Those with schizophrenia were less likely to receive surgical or endoscopic treatment than the controls - even after adjusting for cancer stage. In-hospital mortality within 30 days was significantly higher in patients with schizophrenia than the controls - even after adjusting for cancer stage and undergoing surgical or endoscopic treatment.

\section{Comparisons with other studies}

Advanced cancer stage on first admission in the patients with schizophrenia may reflect a delay in diagnosis or a delay in initiating treatment after diagnosis. Several previous studies with

\begin{tabular}{|c|c|c|c|}
\hline & Case group $(n=2495)$ & Control group $(n=9980)$ & $P$ \\
\hline Cancer stage, $n$ (\%) & & & $<0.001$ \\
\hline । & $595(23.8)$ & $4347(43.6)$ & \\
\hline$\|$ & $402(16.1)$ & $1476(14.8)$ & \\
\hline III & $483(19.4)$ & $1622(16.3)$ & \\
\hline IV & 845 (33.9) & $1805(18.1)$ & \\
\hline Missing & $170(6.8)$ & $730(7.3)$ & \\
\hline Undergoing surgical or endoscopic treatment, $n$ (\%) & $1409(56.5)$ & $7002(70.2)$ & $<0.001$ \\
\hline Open surgery & $848(34.0)$ & $2948(29.5)$ & $<0.001$ \\
\hline Laparoscopic surgery & $363(14.5)$ & $1832(18.4)$ & \\
\hline Endoscopic treatment & $198(7.9)$ & $2222(22.3)$ & \\
\hline Length of stay, days: median & 25 & 15 & \\
\hline 30-day mortality, $n$ (\%) & $104(4.2)$ & $175(1.8)$ & $<0.001$ \\
\hline
\end{tabular}




\begin{tabular}{|c|c|c|}
\hline & Relative risk $(95 \% \mathrm{Cl})$ & $P$ \\
\hline \multicolumn{3}{|l|}{ Group } \\
\hline Control & 1.00 & \\
\hline Case & $1.86(1.72-2.00)$ & $<0.001$ \\
\hline \multicolumn{3}{|l|}{ Gender } \\
\hline Male & 1.00 & \\
\hline Female & $1.10(1.02-1.18)$ & 0.012 \\
\hline \multicolumn{3}{|l|}{ Age, years } \\
\hline $40-59$ & 1.00 & \\
\hline $60-69$ & $1.03(0.89-1.20)$ & 0.673 \\
\hline 70-79 & $0.91(0.79-1.06)$ & 0.240 \\
\hline$\geqslant 80$ & $1.06(0.90-1.25)$ & 0.497 \\
\hline \multicolumn{3}{|c|}{ Charlson comorbidity index score } \\
\hline$\leqslant 2$ & 1.00 & \\
\hline $3-4$ & $0.96(0.89-1.04)$ & 0.280 \\
\hline$\geqslant 5$ & $3.61(3.30-3.95)$ & $<0.001$ \\
\hline \multicolumn{3}{|c|}{$\begin{array}{l}\text { Average neighbourhood } \\
\text { income, thousand US dollars }\end{array}$} \\
\hline$<10.8$ & 1.00 & \\
\hline $10.8-12.2$ & $1.00(0.89-1.12)$ & 0.970 \\
\hline $12.3-13.5$ & $1.00(0.90-1.12)$ & 0.931 \\
\hline $13.6-15.8$ & $1.14(1.02-1.28)$ & 0.027 \\
\hline$>15.8$ & $1.27(1.14-1.43)$ & $<0.001$ \\
\hline \multicolumn{3}{|l|}{ Smoking status } \\
\hline Non-smoker & 1.00 & \\
\hline Smoker & $0.99(0.92-1.07)$ & 0.823 \\
\hline Unknown & $1.27(1.14-1.42)$ & $<0.001$ \\
\hline \multicolumn{3}{|l|}{ Type of cancer } \\
\hline Gastric cancer & 1.00 & \\
\hline Colorectal cancer & $1.74(1.63-1.86)$ & $<0.001$ \\
\hline
\end{tabular}

small sample sizes have reported delayed cancer diagnosis among patients with psychiatric disorders: there was an increased proportion of diagnosis at autopsy ${ }^{35}$ and having metastasis on presentation, ${ }^{18,28}$ as well as suggested delays in initiating treatment after diagnosis. ${ }^{18}$ Other limited studies have reported a reduced likelihood of patients with psychiatric illness undergoing cancer treatment. ${ }^{18,28,35}$ Those studies lacked detailed clinical data, including cancer stage and comorbidities. The present study involved a national large-scale database, which included detailed patient characteristics. Several studies estimated excess cancer mortality in patients with mental illness; ${ }^{18,20}$ however, to our knowledge, no study has examined short-term mortality in in-patients with cancer who also have schizophrenia. Our study selected gastrointestinal cancer because it is highly prevalent in Japan, and it can be detected and treated at an earlier stage than other types of cancer. Our results demonstrated that patients with schizophrenia had higher 30-day in-hospital mortality than nonpsychiatric patients - even after adjustment for cancer stage on admission, undergoing curative treatments and comorbidities.

\section{Interpretation of our findings}

The more advanced cancer stage on admission reflects impaired access to cancer care in patients with schizophrenia. The lower proportion receiving treatment suggests poorer quality of cancer care in this group. A possible explanation for the greater length of stay observed among this group is that these individuals had a more advanced cancer stage and were more likely to undergo open surgery than less invasive treatment, such as laparoscopic surgery and endoscopic treatment. Another explanation is that because the patients with schizophrenia had more comorbidities than the non-psychiatric control group, longer stays were needed after cancer treatment. The higher in-hospital mortality observed

\begin{tabular}{|c|c|c|}
\hline & Odds ratio $(95 \% \mathrm{Cl})$ & $P$ \\
\hline \multicolumn{3}{|l|}{ Group } \\
\hline Control & 1.00 & \\
\hline Case & $0.77(0.69-0.85)$ & $<0.001$ \\
\hline \multicolumn{3}{|l|}{ Gender } \\
\hline Male & 1.00 & \\
\hline Female & $0.98(0.88-1.09)$ & 0.678 \\
\hline \multicolumn{3}{|l|}{ Age, years } \\
\hline $40-59$ & 1.00 & \\
\hline $60-69$ & $1.09(0.88-1.35)$ & 0.447 \\
\hline 70-79 & $1.22(0.98-1.51)$ & 0.069 \\
\hline$\geqslant 80$ & $1.01(0.80-1.27)$ & 0.953 \\
\hline \multicolumn{3}{|c|}{ Charlson comorbidity index score } \\
\hline$\leqslant 2$ & 1.00 & \\
\hline $3-4$ & $1.15(1.03-1.28)$ & 0.016 \\
\hline$\geqslant 5$ & $0.73(0.65-0.82)$ & $<0.001$ \\
\hline \multicolumn{3}{|c|}{$\begin{array}{l}\text { Average neighbourhood } \\
\text { income, thousand US dollars }\end{array}$} \\
\hline$<10.8$ & 1.00 & \\
\hline $10.8-12.2$ & $1.03(0.87-1.21)$ & 0.743 \\
\hline $12.3-13.5$ & $1.09(0.93-1.28)$ & 0.303 \\
\hline $13.6-15.8$ & $1.03(0.87-1.21)$ & 0.760 \\
\hline$>15.8$ & $0.96(0.81-1.13)$ & 0.598 \\
\hline \multicolumn{3}{|l|}{ Smoking status } \\
\hline Non-smoker & 1.00 & \\
\hline Smoker & $1.17(1.05-1.31)$ & 0.005 \\
\hline Unknown & $0.93(0.80-1.09)$ & 0.381 \\
\hline \multicolumn{3}{|l|}{ Type of cancer } \\
\hline Gastric cancer & 1.00 & \\
\hline Colorectal cancer & $1.36(1.23-1.52)$ & $<0.001$ \\
\hline \multicolumn{3}{|l|}{ Cancer stage } \\
\hline I & 1.00 & \\
\hline$\|$ & $0.64(0.54-0.75)$ & $<0.001$ \\
\hline III & $0.47(0.41-0.54)$ & $<0.001$ \\
\hline IV & $0.06(0.06-0.07)$ & $<0.001$ \\
\hline Missing & $0.03(0.03-0.04)$ & $<0.001$ \\
\hline
\end{tabular}

in this study may have resulted not only from impaired access to and low-quality of cancer care, but also unmeasured physical factors in the patients with schizophrenia. Higher mortality was observed both in those individuals with schizophrenia whose cancer was at an early and at an advanced stage. The causes of death should differ between those patients with stage I cancer and those with stage IV cancer. We believe that patients with early-stage cancer would not have died only because of the cancer prognosis within 30 days after admission; their deaths would probably also be related to adverse treatment outcome. Limited studies have reported higher operative morbidity and mortality among patients with psychiatric conditions..$^{29,36,37}$ Factors suggested as affecting poorer operative outcomes in those with psychiatric illness included the following: the patients' psychotic or hazardous health behaviour; interactions between antipsychotics and anaesthetics or analgesias; ${ }^{37}$ and insufficient nutrition. ${ }^{38}$ However, we could not assess the overall short-term mortality because our data did not include post-discharge information.

It should be noted that in this study, the difference in the proportions of patients undergoing surgical or endoscopic treatment between the schizophrenia and control groups were greater for patients with stage IV cancer than for those at other stages. These results indicate that physicians may have tended to avoid invasive treatments for advanced cancer, especially for patients with psychiatric conditions.

Japan has a system of universal health coverage with public health insurance. In principle, insured people may visit a doctor 
Table 5 Multivariable binary logistic regression analysis for 30-day mortality

Odds ratio $(95 \% \mathrm{Cl})$

$P$

Group

Control

1.00

Case

Gender
Male

Female

Age, years

40-59

60-69

70-79

$\geqslant 80$

$1.35(1.04-1.75)$

1.00

$0.95(0.71-1.28)$

0.756

Charlson comorbidity index score

$\leqslant 2$

$3-4$

1.00

$0.94(0.56-1.60)$

$1.16(0.69-1.95)$

0.829

$1.92(1.11-3.31)$

0.020

$\geqslant 5$

1.00

$0.75(0.49-1.15)$

0.181

$2.37(1.72-3.26)$

$<0.001$

Average neighbourhood

income, thousand US dollars

$<10.8 \quad 1.00$

$10.8-12.2$

$12.3-13.5$

$1.22(0.77-1.92)$

$1.71(1.11-2.63)$

0.403

$13.6-15.8$

$1.48(0.96-2.27)$

0.014

$1.10(0.71-1.71)$

0.075

$>15.8$

0.667

Smoking status

Non-smoker

Smoker

1.00

Unknown

$0.83(0.60-1.16)$

0.282

$1.52(1.08-2.14)$

0.016

Type of cancer

Gastric cancer

1.00

Colorectal cancer

$0.63(0.49-0.83)$

0.001

Cancer stage

I 1.00

॥

III

IV

Data missing

$1.55(0.62-3.89)$

0.350

$2.77(1.30-5.92)$

0.008

$5.77(3.03-11.01) \quad<0.001$

Surgical or endoscopic treatment

No $\quad 1.00$

Endoscopic

Open

Laparoscopic

$3.28(1.61-6.67)$

$<0.001$

Reason for admission

Treatment for cancer

Treatment for schizophrenia

Other

$0.06(0.01-0.27)$

$<0.001$

$0.13(0.08-0.22) \quad<0.001$

$0.02(0.00-0.17) \quad<0.001$

1.00

$2.92(0.30-28.07)$

0.354

$1.50(0.99-2.28)$

0.055

at any clinic or hospital in the country. Patients' ability to pay thus has little influence on hospital access or length of stay; however, we observed impaired access to cancer care among patients with schizophrenia in Japan. The present results may not be generalised to other types of patients, such as those with other types of cancer.

\section{Strength and limitations of the study}

The strength of the present study is the use of a large data-set from a national in-patient database. This study is the first to compare cancer stage, treatment and mortality between patients with schizophrenia and non-psychiatric controls by means of detailed clinical information in a nationwide clinical setting.

This study has several limitations. First, the coded diagnoses in the administrative claims database are less well validated than those in prospective studies. Second, because the sample selection was not random, the study participants might have less advanced cancer than all the cancer patients with schizophrenia in Japan, resulting in underestimation of the potential disparity in treatments.
Third, some factors that can affect treatment outcome were not included in our model. In the administrative claims database, we were unable to assess the severity of schizophrenia, which has been reported to affect surgical outcome. ${ }^{29}$ The psychological condition of the patients with schizophrenia during treatment was also unknown. However, it may be assumed that the condition in the majority of those with schizophrenia in the present study was chronic, not acute, because admission to hospital in most was because of the cancer, not schizophrenia (Table 1). We were unable to assess social support and alcohol or substance use, which could be related to survival in cancer patients. Psychotropic medication was not included in our model. We did not include obesity owing to missing data or possible underreporting. Another limitation was lack of information about the cause of death for all the study participants. However, we may infer that many of the deaths were related to adverse treatment outcome or cancer prognosis since we observed deaths only within 30 days of admission to hospital. Finally, the database is de-identified and cannot be linked to other databases at an individual-patient level.

\section{Implications}

Compared with non-psychiatric patients, individuals with schizophrenia with gastrointestinal cancer were admitted to hospital with more advanced cancer, were less likely to undergo invasive treatments and were more likely to die. These results indicate the necessity for improving access to and quality of cancer care for patients with schizophrenia.

Hanako Ishikawa, MD, MPH, Department of Mental Health, Graduate School of Medicine, The University of Tokyo, Tokyo, Japan; Hideo Yasunaga, MD, PhD, Hirok Matsui, MPH, Department of Clinical Epidemiology and Health Economics, School of Public Health, The University of Tokyo, Tokyo, Japan; Kiyohide Fushimi, MD, PhD, Department of Health Policy and Informatics, Tokyo Medical and Dental University Graduate School of Medicine, Tokyo, Japan; Norito Kawakami, MD, PhD,

Department of Mental Health, Graduate School of Medicine, The University of Tokyo,

Tokyo, Japan

Correspondence: Hideo Yasunaga, MD, PhD, Department of Clinical

Epidemiology and Health Economics, School of Public Health, The University of Tokyo, 7-3-1 Hongo, Bunkyo-ku, Tokyo 1130033, Japan. Email: yasunagah-tky@ umin.ac.jp

First received 13 Aug 2014, final revision 3 Apr 2015, accepted 15 Apr 2015

\section{Funding}

This study was funded by a grant-in-aid from the Ministry of Health, Labour and Welfare, Japan (grant number: H26-Policy-011). The funder had no role in study design, data collection and analysis, decision to publish or preparation of the manuscript.

\section{References}

1 Harris EC, Barraclough B. Excess mortality of mental disorder. Br J Psychiatry 1998; 173: 11-53.

2 Brown S. Excess mortality of schizophrenia. A meta-analysis. Br J Psychiatry 1997; 171: 502-8.

3 Brown S, Kim M, Mitchell C, Inskip H. Twenty-five year mortality of a community cohort with schizophrenia. Br J Psychiatry 2010; 196: 116-21.

4 Saha S, Chant D, McGrath J. A systematic review of mortality in schizophrenia: is the differential mortality gap worsening over time? Arch Gen Psychiatry 2007; 64: 1123-31.

5 Lawrence D, Hancock KJ, Kisely S. The gap in life expectancy from preventable physical illness in psychiatric patients in Western Australia: retrospective analysis of population based registers. BMJ 2013; 346: f2539.

6 Joukamaa M, Heliövaara M, Knekt $\mathrm{P}$, Aromaa A, Raitasalo R, Lehtinen V. Mental disorders and cause-specific mortality. Br J Psychiatry 2001; 179: 498-502.

7 Brown S, Inskip H, Barraclough B. Causes of the excess mortality of schizophrenia. Br J Psychiatry 2000; 177: 212-7. 
8 Lawrence D, Jablensky AV, Holman CD, Pinder TJ. Mortality in Western Australian psychiatric patients. Soc Psychiatry Psychiatr Epidemiol 2000; 35 341-7.

9 Osby U, Brandt L, Correia N, Ekbom A, Sparen P. Excess mortality in bipolar and unipolar disorder in Sweden. Arch Gen Psychiatry 2001; 58: 844-50.

10 Tran E, Rouillon F, Loze JY, Casadebaig F, Philippe A, Vitry F, et al. Cancer mortality in patients with schizophrenia: an 11-year prospective cohort study. Cancer 2009; 115: 3555-62.

11 Leucht S, Burkard T, Henderson J, Maj M, Sartorius N. Physical illness and schizophrenia: a review of the literature. Acta Psychiatr Scand 2007; 116: 317-33.

12 Lichtermann D, Ekelund J, Pukkala E, Tanskanen A, Lonnqvist J. Incidence of cancer among persons with schizophrenia and their relatives. Arch Gen Psychiatry 2001; 58: 573-8.

13 Barak Y, Achiron A, Mandel M, Mirecki I, Aizenberg D. Reduced cancer incidence among patients with schizophrenia. Cancer 2005; 104: 2817-21.

14 Catts VS, Catts SV, O'Toole BI, Frost AD. Cancer incidence in patients with schizophrenia and their first-degree relatives - a meta-analysis. Acta Psychiatr Scand 2008; 117: 323-36.

15 Gal G, Goral A, Murad H, Gross R, Pugachova I, Barchana M, et al. Cancer in parents of persons with schizophrenia: is there a genetic protection? Schizophr Res 2012; 139: 189-93.

16 Goldacre MJ, Kurina LM, Wotton CJ, Yeates D, Seagroat V. Schizophrenia and cancer: an epidemiological study. Br J Psychiatry 2005; 187: 334-8.

17 Dalton So, Laursen TM, Mellemkjaer L, Johansen C, Mortensen PB. Risk for cancer in parents of patients with schizophrenia. Am J Psychiatry 2004; 161: 903-8.

18 Kisely S, Crowe E, Lawrence D. Cancer-related mortality in people with mental illness. JAMA Psychiatry 2013; 70: 209-17.

19 Kisely S, Sadek J, MacKenzie A, Lawrence D, Campbell LA. Excess cancer mortality in psychiatric patients. Can J Psychiatry 2008; 53: 753-61.

20 Lawrence D, Holman CD, Jablensky AV, Threlfall TJ, Fuller SA. Excess cance mortality in Western Australian psychiatric patients due to higher case fatality rates. Acta Psychiatr Scand 2000; 101: 382-8.

21 Crump C, Winkleby MA, Sundquist K, Sundquist J. Comorbidities and mortality in persons with schizophrenia: a Swedish national cohort study. Am J Psychiatry 2013; 170: 324-33.

22 Irwin KE, Henderson DC, Knight HP, Pirl WF. Cancer care for individuals with schizophrenia. Cancer 2014; 120: 323-34.

23 Aggarwal A, Pandurangi A, Smith W. Disparities in breast and cervical cancer screening in women with mental illness: a systematic literature review. Am J Prev Med 2013; 44: 392-8.
24 Chochinov HM, Martens PJ, Prior HJ, Fransoo R, Burland E. Does a diagnosis of schizophrenia reduce rates of mammography screening? A Manitoba population-based study. Schizophr Res 2009; 113: 95-100.

25 Carney $\mathrm{CP}$, Jones LE. The influence of type and severity of mental illness on receipt of screening mammography. J Gen Intern Med 2006; 21: 1097-104.

26 Xiong GL, Bermudes RA, Torres SN, Hales RE. Use of cancer-screening services among persons with serious mental illness in Sacramento County. Psychiatr Serv 2008; 59: 929-32.

27 Farasatpour M, Janardhan R, Williams CD, Margenthaler JA, Virgo KS, Johnson FE. Breast cancer in patients with schizophrenia. Am J Surg 2013; 206: 798-804.

28 O'Rourke RW, Diggs BS, Spight DH, Robinson J, Elder KA, Andrus J, et al. Psychiatric illness delays diagnosis of esophageal cancer. Dis Esophagus 2008; 21: 416-21.

29 Liao CC, Shen WW, Chang CC, Chang H, Chen TL. Surgical adverse outcomes in patients with schizophrenia: a population-based study. Ann Surg 2013; 257: 433-8

30 Yasunaga $\mathrm{H}$, Horiguchi $\mathrm{H}$, Kuwabara $\mathrm{K}$, Matsuda S, Fushimi $\mathrm{K}$, Hashimoto $\mathrm{H}_{\text {, }}$ et al. Outcomes after laparoscopic or open distal gastrectomy for early-stage gastric cancer: a propensity-matched analysis. Ann Surg 2013; 257: 640-6.

31 Yasunaga $H$, Hashimoto $H$, Horiguchi $H$, Miyata $H$, Matsuda S. Variation in cancer surgical outcomes associated with physician and nurse staffing: a retrospective observational study using the Japanese Diagnosis Procedure Combination Database. BMC Health Serv Res 2012; 12: 129.

32 World Health Organization. ICD-10: International Statistical Classification of Diseases and Related Health Problems, Tenth Revision. WHO, 1992.

33 Quan H, Sundararajan V, Halfon P, Fong A, Burnand B, Luthi JC, et al. Coding algorithms for defining comorbidities in ICD-9-CM and ICD-10 administrative data. Med Care 2005; 43: 1130-9.

34 Charlson ME, Pompei P, Ales KL, MacKenzie CR. A new method of classifying prognostic comorbidity in longitudinal studies: development and validation. J Chronic Dis 1987; 40: 373-83.

35 Baillargeon J, Kuo YF, Lin YL, Raji MA, Singh A, Goodwin JS. Effect of mental disorders on diagnosis, treatment, and survival of older adults with colon cancer. J Am Geriatr Soc 2011; 59: 1268-73.

36 Copeland LA, Zeber JE, Pugh MJ, Mortensen EM, Restrepo MI, Lawrence VA. Postoperative complications in the seriously mentally ill: a systematic review of the literature. Ann Surg 2008; 248: 31-8.

37 Kudoh A. Perioperative management for chronic schizophrenic patients. Anesth Analg 2005; 101: 1867-72.

38 Hashimoto N, Isaka N, Ishizawa Y, Mitsui T, Sasaki M. Surgical management of colorectal cancer in patients with psychiatric disorders. Surg Today 2009; 39: 393-8. 